Aim The aim of this study was to determine the incidence of (Healthcare-associated infection) HAI, causative organisms, associated risk factors in a neonatal intensive care unit in Turkey.

Methods A prospective cohort study was conducted on patients admitted to the neonatal intensive care unit (NICU) from July 2011 to June 2012. The criteria that were used to diagnose infection were in accordance with the Centres for Disease Control and Prevention. The incidence, causative organisms, risk factors and mortality of healthcare-associated infections were assessed.

Results The study included 352 patients, 37 of these developed HAIs, totaling $60 \mathrm{HAI}$ episodes. Overall HAI patient rate was $17.04 \%$, and 11.51 HAIs per 1000 NICU days. The most frequent HAIs were bloodstream infections (70\%) and nosocomial pneumonia (18.3\%). The central venous catheter/umblical catheter-related bloodstream infections (CVC/UC BSIs) rate was 18.3/ 1,000 catheter days; the ventilator-associated pneumonia (VAP) rate was 13.6/1,000 ventilator days; and the catheter-associated urinary tract infections rate found was 14.9/1,000 catheter days. Prematurity, gestational age less than 32 weeks, birth weight $<$ $1500 \mathrm{~g}$, mechanical ventilation, use of CVC/UC, use of urinary catheter, and total parenteral nutrition appeared to be associated with a significantly higher risk of HAI ( $\mathrm{p} \leq 0.05)$. The most frequent pathogens were Enterobacter spp. (18.5\%) and Acinetobacter baumannii (13.8\%). Overall mortality rate in neonates was $3.9 \%$, and the mortality rate in neonates with HAI was $10.8 \%$.

Conclusions Healthcare-associated infection rates of our NICU were higher than international standards. The decrement of risk factors in newborns would help to improve the outcome.

\section{PO-0517 FIRST REPORTED CASE OF REPEATED SUBCUTANEOUS ADMINISTRATION OF PALIVIZUMAB IN A FORMER PRETERM INFANT}

${ }^{1} \mathrm{~K}$ Allegaert, ${ }^{1} \mathrm{~V}$ Cossey, ${ }^{1} \mathrm{M}$ Rayyan, ${ }^{2} \mathrm{C}$ Van Geet. ${ }^{1}$ Neonatal Intensive Care Unit, University Hospitals Leuven, Leuven, Belgium; ${ }^{2}$ Pediatrics, University Hospitals Leuven, Leuven, Belgium

\subsection{6/archdischild-2014-307384.1161}

Background and aims Intramuscular injection is contra-indicated in patients with bleeding disorders. In these cases, the unlicensed practice is to administer vaccines by subcutaneous route. Despite extensive literature search and communication with the manufacturer, we were unable to retrieve any reported experience with palivizumab. Supported by a mathematical description (Zhao L et al. J Clin Pharmacol 2013) for subcutaneous or intramuscular administration of monoclonal antibodies, we decided to administer pavilizumab by subcutaneous route and to document the effects. Parental informed consent was obtained.

Methods Prospective registration of tolerance and effects of subcutaneous palivizumab administration in a former preterm girl with von Willebrand, type 2B. Spare plasma samples (peak and through levels) were also collected, but analysis was not possible since we had no access to a valid quantification technique.

Results The girl received 5 monthly subcutaneous injections (15 $\mathrm{mg} / \mathrm{kg}$ ) and was subsequently monitored for $1 \mathrm{~h}$ for systemic and local side effects with further evaluation of tolerance by the parents. Local tolerance of palivizumab (tenderness, swelling) was much better when compared to the simultaneously administered (Infarix Hexa, or Prevenar subcutaneous) vaccines. She never displayed RSV-related symptoms.
Conclusions Repeated subcutaneous injection of palivizumab was tolerated well with minor local reactions and no systemic side effects. We suggest to consider the subcutaneous instead of the intramuscular route in the setting of a valid indication for palivizumab, but an contraindication for intramuscular administration. Off-label or unlicensed practices should be reported to share and improve knowledge on pharmacotherapy.

\section{PO-0518 THE INCIDENCE AND THE MICROBIAL PATTERN OF NEONATAL SEPSIS IN JORDAN}

A Almatti. Neonatology - and Pediatrics, Islamic Hospital, Amman, Jordan

\subsection{6/archdischild-2014-307384.1162}

Introduction Neonatal sepsis is a common and potentially serious neonatal disease especially in preterm babies and more complicated in developing countries.

Objectives To study the incidence and the microbial pattern of neonatal sepsis in our unit in Amman/Jordan.

Method A retrospective study of all newborn cases admitted to our NICU as suspected sepsis over a 4-year period, 2001-2004 analysing the results of blood cultures.

Results The total number of newborns delivered in our NICU over a 4-year period was 25715 .

- 819 (3.2\%) babies were admitted as suspected sepsis. *616 (75\%) were full term babies. *203 (25\%) were preterm babies (28 to 36 weeks)

- The total culture- proven cases (positive blood cultures) were 51 (6.2\%).out of which $21(40 \%)$ cases were preterm babies. So culture-proven sepsis was found in about $10 \%$ of preterm babies and in about $5 \%$ of full term babies who were admitted as suspected sepsis.

- The overall incidence of sepsis was 2 per 1000.

- Gram negative organisms recovered in 32 cases (63\%), mainly Klebsiella species in 21 cases (40\%), E.coli in 7(14\%)and Pseudomonas in 4 cases (8\%).

- Gram positive cases were 19(37\%) : Staphylococci aureus in 7 cases, Staph. Epidermidis in 4, GBS in 5 and Strept.viridans in 3 cases.

The mortality among all culture-proven cases was 2 cases $(4 \%)$.

Conclusion - A bout 3.2\% of all newborns were admitted as suspected sepsis.

- Only 6\% of suspected cases of sepsis were proven culture positive-The overall incidence of neonatal sepsis in our unit is 2 / 1000 .

- Klebsiella species is the commonest pathogen isolated in our NICU.

\section{P0-0519 CAN BASE EXCESS BE USED FOR EARLY DIAGNOSIS OF NEONATAL SEPSIS IN PRETERM NEWBORNS?}

${ }^{1} \mathrm{~S}$ Arayici, ${ }^{1} \mathrm{G}$ Kadioglu Simsek, ${ }^{1} \mathrm{FE}$ Canpolat, 'M Oncel, ${ }^{1} \mathrm{~N}$ Uras, 'S Oguz, ${ }^{2} \mathrm{U}$ Dilmen. ${ }^{1}$ Neonatology, Zekai Tahir Burak Maternity Teaching Hospital, Ankara, Turkey; ${ }^{2}$ Neonatology, Yildirim Beyazit University School of Medicine, Ankara, Turkey

\subsection{6/archdischild-2014-307384.1163}

Background and aims Neonatal sepsis remains an important and potentially life-threatening clinical syndrome and a major cause of neonatal mortality and morbidity, particularly in preterm infants. The aim of this study to investigate whether values of 